\title{
Some Finiteness Conditions for Strong Semilattice of Semigroups
}

\section{Basri Çalişkan}

Department of Mathematics, Faculty of Arts and Science, Osmaniye Korkut Ata University, Osmaniye, Turkey

\section{Email address:}

bcaliskan@osmaniye.edu.tr

\section{To cite this article:}

Basri Çalişkan. Some Finiteness Conditions for Strong Semilattice of Semigroups. Pure and Applied Mathematics Journal. Vol. 6, No. 6, 2017, pp. 160-163. doi: 10.11648/j.pamj.20170606.12

Received: October 13, 2017; Accepted: October 31, 2017; Published: December 18, 2017

\begin{abstract}
Let $S=\mathcal{S}\left[I ; S_{i}, \phi_{j, i}\right]$ be a strong semilattice of semigroups such that $I$ is finite and each $S_{i}(i \in I)$ be a family of disjoint semigroups. In this article some finiteness conditions which are periodicity, local finiteness and locally finite presentability are considered for $S$. It is proven that a strong semilattice of semigroups $\mathcal{S}\left[I ; S_{i}, \phi_{j, i}\right]$ is periodic, locally finite, locally finitely presented and residually finite, respectively if and only if $I$ is finite and each semigroup $S_{i}(i \in I)$ is periodic, locally finite, locally finitely presented and residually finite, respectively.
\end{abstract}

Keywords: Strong Semilattice of Semigroups, Finiteness Conditions, Periodicity, Local Finiteness, Locally Finite Presentability, Residual Finiteness

\section{Introduction}

The study of finiteness conditions for semigroups (the properties of semigroups which all finite semigroups have) is one of the most important topics for the mathematical community. Some of the properties of finiteness are being finitely generated, finite presentability, having a soluble word problem, periodicity, local finiteness, local finite presentability, residual finiteness, being hopfian, having finite complete rewriting systems and finite derivation type (FDT). They have been considered for certain classes of semigroup constructions (see [1-9]). In [1], it was shown that the properties of being finite, being finitely generated, being finitely presented and being residually finite are preserved by large subsemigroups and small extensions. In [5], periodicity, local finiteness, residual fniteness of Rees matrix semigroups and solvable word problem for Rees matrix semigroups were investigated.

One of semigroup structures is a strong semilattice of semigroups which is an important structure for completely regular semigroups (see [10]). Finite presentability of strong semilattice of semigroups was investigated in [3]. The authors of [3] first proved that a band of semigroups $S_{i}$ $(i \in I)$ is finitely presented if $I$ is finite and each $S_{i}$ is finitely presented (see [3, Corollary 4.5]). Moreover, they proved that a band of monoids $S_{i} \quad(i \in I)$ is finitely presented if and only if $I$ is finite and each $S_{i}$ is finitely presented (see [3, Corollary 5.6]). Then, from Theorem 1.3 in [1] they concluded that a strong semilattice of semigroups $\mathcal{S}\left[I ; S_{i}, \phi_{j, i}\right]$ is finitely presented if each $S_{i} \quad(i \in I)$ is finitely presented and if $I$ is finite. In [4] the authors constructed more specific presentations for strong semilattice of semigroups from given presentations for $S_{i}(i \in I)$ and a presentation for each $S_{i}$ from a given presentation for $\mathcal{S}\left[I ; S_{i}, \phi_{j, i}\right]$. Also they proved that if $\mathcal{S}\left[I ; S_{i}, \phi_{j, i}\right]$ is finitely generated then $\mathcal{S}\left[I ; S_{i}, \phi_{j, i}\right]$ has a soluble word problem if and only if each $S_{i}$ has a soluble word problem. In [9] the author showed that a strong semilattice of semigroups $\mathcal{S}\left[I ; S_{i}, \phi_{j, i}\right]$ has FDT if and only if $I$ is finite and every semigroup $S_{i}(i \in I)$ has FDT. 
In this paper periodicity, locally finiteness, locally finite presentability and residual finiteness of strong semilattice of semigroups are investigated.

\section{Notation and Basic Definitions}

Let $\mathcal{P}$ be any property of semigroups. It said that a semigroup is locally $\mathcal{P}$ if every finitely generated subsemigroup of has property $\mathcal{P}$. If $\mathcal{P}$ is a finiteness condition, then being locally $\mathcal{P}$ is also a finiteness condition. In particular, being locally finite and being locally finitely presented are finiteness conditions.

Let $I$ be a semilattice, and let $S_{i}(i \in I)$ be a family of disjoint semigroups. Suppose that, for any two elements $i, j \in I$, with $i \leq j$ ( $i \leq j$ if and only if $i j=i$ ), there is a homomorphism $\phi_{j, i}: S_{j} \rightarrow S_{i} \quad, \quad$ and that these homomorphisms satisfy the following conditions:

1. for each $i \in I, \phi_{i, i}$ is the identity map $1_{S_{i}}$;

2. for all $i, j, k \in I$, such that $i \leq j \leq k \quad \phi_{k, j} \phi_{j, i}=\phi_{k, i}$.

Define a multiplication on $S=\bigcup_{i \in I} S_{i}$ in terms of the multiplications in the components $S_{i}$ and the homomorphisms $\phi_{j, i}$, by the rule that, for each $x \in S_{i}$ and $y \in S_{j}$,

$$
x y=\left(x \phi_{i, i j}\right)\left(y \phi_{j, i j}\right)
$$

With respect to this multiplication, $S$ is a semigroup, called a strong semilattice of semigroups, and is denoted by $\mathcal{S}\left[I ; S_{i}, \phi_{j, i}\right]$.

Let $A$ be an alphabet, $A^{+}$be the free semigroup on $A$ (i.e. the set of all non-empty words over $A$ ). A semigroup presentation is an ordered pair $\langle A \mid R\rangle$ where $R \subseteq A^{+} \times A^{+}$. An element $a$ of $A$ is called a generating symbol, while an element $(r, s)$ of $R$ is called a defining relation, and is usually written as $r=s$. Also if $A=\left\{a_{1}, a_{2}, \ldots, a_{n}\right\}$ and $R=\left\{r_{1}=s_{1}, r_{2}=s_{2}, \ldots, r_{n}=s_{n}\right\} \quad, \quad$ we write $\left\langle a_{1}, a_{2}, \ldots, a_{n} \mid r_{1}=s_{1}, r_{2}=s_{2}, \ldots, r_{n}=s_{n}\right\rangle$ for $\langle A \mid R\rangle$. The semigroup defined by a presentation $\langle A \mid R\rangle$ is $A^{+} / \rho$, where $\rho$ is the least congruence on $A^{+}$containing $R$. More generally, a semigroup $S$ is said to be defined by the presentation $\langle A \mid R\rangle$ if $S \cong A^{+} / \rho$. Thus, elements of $S$ are in one-one correspondence with the congruence classes of words from $A^{+}$, or, to put it differently, each word from $A^{+}$ represents an element of $S$. As usual, for any two words $u_{1}, u_{2} \in A^{+}$we write $u_{1}=u_{2}$ if $u_{1} \rho=u_{2} \rho$ (i.e. if they represent the same element in $S$ ), and $u_{1} \equiv u_{2}$ if they are identical words. The semigroup $S$ is finitely generated if the set $A$ is finite; $S$ is finitely presented if both sets $A$ and $R$ are finite.

\section{Periodicity}

Recall that a semigroup $S$ is periodic if, for each $s \in S$, the monogenic semigroup generated by $s$ is finite, or equivalently there exist distinct positive integers $m$ and $r$ (depending on $s$ ) such that $s^{m+r}=s^{m}$.

Theorem 3.1 Let $I$ be a finite semilattice, and let $S_{i}$ $(i \in I)$ be a family of disjoint semigroups. The strong semilattice $S=\mathcal{S}\left[I ; S_{i}, \phi_{j, i}\right]$ is periodic if and only if, for each $i \in I, S_{i}$ is periodic.

Proof.

$(\Rightarrow)$ Since each $S_{i} \quad(i \in I)$ is a subsemigroup of the strong semilattice $S$ and the subsemigroups of the periodic semigroups are periodic, each semigroup $S_{i} \quad(i \in I)$ is periodic.

$(\Leftarrow)$ Let each $S_{i} \quad(i \in I)$ be periodic. Let $s \in \bigcup_{i \in I} S_{i}$. Then, there exists an $i$ such that $s \in S_{i}$. Since $S_{i}$ is periodic, there exist positive integers $m$ and $r$ such that $s^{m+r}=s^{m}$. So the strong semilattice $S=\mathcal{S}\left[I ; S_{i}, \phi_{j, i}\right]$ is periodic.

\section{Local Finiteness}

Let $X$ be any non-empty subset of a semigroup $S$. Then the smallest subsemigroup of $S$ containing $X$ is called the subsemigroup of $S$ generated by $X$, and is denoted by $\langle X\rangle$. If $X$ is finite then $\langle X\rangle$ is called a finitely generated subsemigroup. If every finitely generated subsemigroup of $S$ is finite, then $S$ is called locally finite.

Theorem 4.1

Let $I$ be a finite semilattice, and let $S_{i} \quad(i \in I)$ be a family of disjoint semigroups. The strong semilattice $S=\mathcal{S}\left[I ; S_{i}, \phi_{j, i}\right]$ is locally finite if and only if, for each $i \in I, S_{i}$ is locally finite.

Proof.

$(\Rightarrow)$ Since each $S_{i} \quad(i \in I)$ is a subsemigroup of the strong semilattice $S$ and the subsemigroups of the locally finite semigroups are locally finite, each semigroup $S_{i}$ $(i \in I)$ is locally finite.

$(\Leftarrow)$ Let each $S_{i} \quad(i \in I)$ be locally finite. Let $X$ be a finite subset of the strong semilattice $S=\mathcal{S}\left[I ; S_{i}, \phi_{j, i}\right]$. Now it is shown that $\langle X\rangle$, the subsemigroup generated by $X$, is finite.

Let $J \subseteq I$ and define $J$ as follows 


$$
i \in J \Leftrightarrow X \cap S_{i} \neq \varnothing \quad(i \in I)
$$

Then, since $X$ is finite, the set $J=\left\{i_{1}, \ldots, i_{n}\right\}$ is finite. Let $X_{i}=X \cap S_{i}$ for each $i \in J$. Then $\left\{X_{i}\right\}_{i \in J}$ is a partition of $X$. Also, for each subset $\left\{j_{1}, \ldots, j_{k}\right\}$ of $J$, there exists a $\lambda$ such that $j_{1} \cdots j_{k} \leq j_{\lambda}$. Then, since $1 \leq \lambda \leq k$, the set $Y_{\left\{j_{1}, \ldots, j_{k}\right\}}$ can be taken as follows,

$$
Y_{\left\{j_{1}, \ldots, j_{k}\right\}}=\bigcup_{\lambda=1}^{k}\left\{x \phi_{j_{\lambda}, j_{1} \cdots j_{k}} \mid x \in X_{j_{\lambda}}\right\}
$$

For each $1 \leq \lambda \leq k$, the set $X_{j_{\lambda}}$ is finite, since the union of finitely many finite sets is finite, and thus the set $Y_{\left\{j_{1}, \ldots, j_{k}\right\}}$ is a finite subset of $S_{j_{1} \cdots j_{k}}$. Since $S_{j_{1} \cdots j_{k}}$ is locally finite, then $\left\langle Y_{\left\{j_{1}, \ldots, j_{k}\right\}}\right\rangle$ is finite. As $J$ has subsets with finitely many numbers, the set

$$
T=\bigcup_{\varnothing \neq\left\{j_{1}, \ldots, j_{k}\right\} \subseteq J}\left\langle Y_{\left\{j_{1}, \ldots, j_{k}\right\}}\right\rangle
$$

is finite.

Now, it is shown that the set $\langle X\rangle$ is finite. For any $s \in\langle X\rangle$, there exists $x_{1} \in X_{j_{1}}, \ldots, x_{m} \in X_{j_{m}}$ such that $s=x_{1} x_{2} \cdots x_{m}$.

Let $K=\left\{j_{1}, j_{2}, \ldots, j_{m}\right\} \quad, \quad$ then $\quad \varnothing \neq K \subseteq J \quad$ and $s \in\left\langle Y_{K}\right\rangle \subseteq T$. So, $\langle X\rangle \subseteq T$ and $\langle X\rangle$ is finite. Hence $S=\mathcal{S}\left[I ; S_{i}, \phi_{j, i}\right]$ is locally finite.

\section{Locally Finite Presentability}

Let $S$ be a semigroup. If every finitely generated subsemigroup of $S$ is finitely presented, then $S$ is called locally finitely presented.

Theorem 5.1 Let $I$ be a finite semilattice, and let $S_{i}$ $(i \in I)$ be a family of disjoint semigroups. The strong semilattice $S=\mathcal{S}\left[I ; S_{i}, \phi_{j, i}\right]$ is locally finitely presented if and only if, for each $i \in I, S_{i}$ is locally finitely presented.

Proof.

$(\Rightarrow)$ Since each $S_{i} \quad(i \in I)$ is a subsemigroup of the strong semilattice $S$ and the subsemigroups of the locally finitely presented semigroups are locally finitely presented, each semigroup $S_{i}(i \in I)$ is locally finitely presented.

$(\Leftarrow)$ Let each $S_{i}(i \in I)$ be locally finitely presented. Let $X$ be a finite subset of the strong semilattice $S=\mathcal{S}\left[I ; S_{i}, \phi_{j, i}\right]$. Let define the set $J$ as follows,

$$
J=\left\{i \in I \mid X \cap S_{i} \neq \varnothing\right\}
$$

Also, let define $p(K) \in I$ for all $\varnothing \neq K \subseteq J$ as

$$
K=\left\{i_{1}, \ldots, i_{m}\right\} \Rightarrow p(K)=i_{1} \cdots i_{m}
$$

Then, since $J$ is finite, the set $P(J) \subseteq I$ that is defined by

$$
P(J)=\{p(K) \mid \varnothing \neq K \subseteq J\}
$$

is finite. It is obvious that $P(J)$ is a subsemigroup of $I$ generated by $J$. So $P(J)$ is a semilattice.

Let $X_{i}=X \cap S_{i}$ be a set for all $i \in J$. Let $K$ be a subset of $J$. Let define $Y_{K}$ as follows

$$
Y_{K}=\bigcup_{i \in K}\left\{x \phi_{i, p(K)} \mid x \in X_{i}\right\}
$$

And for all $k \in P(K), Z_{k}$ can be defined as follows

$$
Z_{k}=\bigcup_{\varnothing \neq K \subseteq J \text { ve } p(K)=k} Y_{K}
$$

Then $Z_{k} \subseteq S_{k}$ for all $k \in P(K)$. Since $S_{k}$ is locally finitely presented, then $T_{k}=\left\langle Z_{k}\right\rangle$ the subsemigroup generated by $Z_{k}$ is finitely presented.

Now it is shown that the subsemigroup $\langle X\rangle$ is finitely presented. For any $s \in\langle X\rangle$, there exists

$x_{1} \in X_{j_{1}}, \ldots, x_{m} \in X_{j_{m}}$ such that $s=x_{1} x_{2} \cdots x_{m}$.

Let $K_{s}=\left\{j_{1}, j_{2}, \ldots, j_{m}\right\}$. If $p\left(K_{s}\right)=k_{s}$, then $s \in T_{k_{s}}$. Also from the definition of $T_{k}, T_{k} \subseteq\langle X\rangle$. Since each $S_{k}$ is disjoint, then each $T_{k}$ is a disjoint subsemigroup of $\langle X\rangle$ and $P(J)$ is finite, and so $\langle X\rangle$ is the union of the finitely many finitely presented disjoint semigroups $T_{k}$, that is,

$$
\langle X\rangle=\bigcup_{k \in P(J)} T_{k}
$$

Finally, let $\varphi_{k, i}$ be the restriction of the homomorphisms of $\phi_{k, i}$ to the subsemigroup $T_{k}=\left\langle Z_{k}\right\rangle$. So it is clear that the strong semilattice $\mathcal{S}\left[P(J) ; T_{k}, \varphi_{k, i}\right]$. Hence from Theorem 1 in [4], $\langle X\rangle$ is finitely presented.

\section{Residual Finiteness}

Residual finiteness is one of the most important algebraic finiteness conditions, and has been widely studied in the context of groups, semigroups, monoids and other algebraic structures. Let $X$ be a set and let $\pi$ be an equivalence relation 
on $X$. For $x \in X$ we use $x / \pi$ to denote the equivalence class of the element $x$, and $X / \pi$ to denote the set of all equivalence classes. The number of $\pi$-classes of $X$ is denoted $[X: \pi]$ and called this the index of the relation $\pi$. It is said that $\pi$ separates the elements $s$ and $t$ if $s / \pi=t / \pi$. A semigroup $S$ is residually finite if for every two distinct $x, y \in S$ there is a congruence $\rho$ on $S$ which has finite index and which separates $x$ and $y$. This is equivalent to saying that there is a homomorphism $\Phi$ from $S$ onto a finite semigroup such that $\Phi(s) \neq \Phi(t)$.

\section{Theorem 6.1}

Let $I$ be a finite semilattice, and let $S_{i} \quad(i \in I)$ be a family of disjoint semigroups. The strong semilattice $S=\mathcal{S}\left[I ; S_{i}, \phi_{j, i}\right]$ is residually finite if and only if, for each $i \in I, S_{i}$ is residually finite.

Proof.

$(\Rightarrow)$ Since each $S_{i} \quad(i \in I)$ is a subsemigroup of the strong semilattice $S$ and the subsemigroups of the residually finite semigroups are residually finite, each semigroup $S_{i}$ $(i \in I)$ is residually finite.

$(\Leftarrow)$ Suppose that each semigroup $S_{i} \quad(i \in I)$ is residually finite. Then, for all $i \in I$, there exist homomorphisms $\sigma_{i}$ from $S_{i}$ onto a finite semigroup $S_{i}^{\prime}$ such that $\sigma_{i}\left(w_{i_{k}}\right) \neq \sigma_{i}\left(w_{i_{l}}\right)$ for each $w_{i_{k}}, w_{i_{l}} \in S_{i}$.

Let $w_{i_{k}}$ and $w_{i_{l}}$ be two different elements in the strong semilattice $S=\mathcal{S}\left[I ; S_{i}, \phi_{j, i}\right]$. If $w_{i_{k}}$ and $w_{i_{l}}$ are both in the same subsemigroup $S_{i}$ of $S$, then there exists a finite semigroup $S^{\prime}=\bigcup_{i \in I} S_{i}^{\prime}$ and a homomorphism $\varphi\left(w_{i_{k}}\right)=\sigma_{i}\left(w_{i_{k}}\right)$. It is clear that $\varphi$ is well-defined onto homomorphism from $S$ to $S^{\prime}$ such that $\varphi\left(w_{i_{k}}\right)=\sigma_{i}\left(w_{i_{k}}\right) \neq \sigma_{i}\left(w_{i_{l}}\right)=\varphi\left(w_{i_{l}}\right)$.

If they are not in the same subsemigroup of the strong semilattice $S=\mathcal{S}\left[I ; S_{i}, \phi_{j, i}\right]$. Then both of them are distinct elements. Assume that $w_{i_{n}} \in S_{i}$ and $w_{j_{m}} \in S_{j}$. Since $S_{i}$ and $S_{j}$ are residually finite, there are finite semigroups $S_{i}^{\prime}, S_{j}^{\prime}$ and homomorphisms $\sigma_{i}: S_{i} \rightarrow S_{i}^{\prime}, \sigma_{j}: S_{j} \rightarrow S_{j}^{\prime}$.

If the mapping $\psi$ is defined from the strong semilattice $S=\mathcal{S}\left[I ; S_{i}, \phi_{j, i}\right]$ to the finite semigroup $S^{\prime}=\bigcup_{i \in I} S_{i}^{\prime}$ as follows,

$$
\psi\left(w_{i_{n}}\right)= \begin{cases}\sigma_{i}\left(w_{i_{n}}\right), & w_{i_{n}} \in S_{i}(i \in I) \\ \sigma_{j}\left(w_{i_{n}}\right), & w_{i_{n}} \in S_{j}(j \in I)\end{cases}
$$

Since $\sigma_{i}$ is onto, it is clear that $\psi$ is well-defined. Thus it follows that $\psi: S \rightarrow S^{\prime}$ is an onto homomorphism such that $\psi\left(w_{i_{n}}\right) \neq \psi\left(w_{i_{m}}\right)$. Thus, the strong semilattice $S=\mathcal{S}\left[I ; S_{i}, \phi_{j, i}\right]$ is residually finite.

\section{Conclusion}

One of semigroup structures is a strong semilattice of semigroups which is an important structure for completely regular semigroups.

In this article it is proven that a strong semilattice of semigroups $\mathcal{S}\left[I ; S_{i}, \phi_{j, i}\right]$ is periodic, local finite, locally finitely presented and residually finite, respectively if and only if $I$ is finite and each semigroup $S_{i}(i \in I)$ is periodic, locally finite, locally finitely presented and residually finite, respectively.

\section{References}

[1] N. Ruskuc, "On large subsemigroups and finiteness conditions of semigroups,” Proc. London Math. Soc., vol. 76, 1998, pp. 383-405.

[2] H. Ayık, N. Ruskuc, "Generators and relations of Rees matrix semigroups," Proc. Edinburgh Math. Soc., vol. 42, 1999, pp. 481-495.

[3] I. M. Araujo, M. J. J. Branco, V. H. Fernandes, G. M. S. Gomes, N. Ruskuc, "On generators and relations of unions of semigroups,” Semigroup Forum, vol. 63, 2001, pp. 49-62.

[4] G. Ayık, H. Ayık, Y. Ünlü, "Presentations and word problem for strong semilattices of semigroups," Algebra and Discrete Mathematics, vol. 4, 2005, pp. 1-8.

[5] H. Ay1k, "On finiteness conditions for Rees matrix semigroups," Czechoslovak Math. J., vol. 55, 2005, pp. 455463.

[6] B. Çalışkan, "On finitness conditions for $S, \rho$ and $S / \rho$," International Journal of Pure and Applied Mathematics, vol. 65 (1), 2010, pp. 1-9.

[7] R. Gray, N. Ruskuc, "On residual finiteness of monoids, their Schützenberger groups and associated actions", J. Algebra 407 (2014), 21-45. DOI:10.1016/j.jalgebra.2014.02.025 arXiv: 1003.3176 .

[8] I. Dolinka, R. Gray, N. Ruskuc, On regularity and the word problem for free idempotent generated semigroups, Proc. London Math. Soc. 114 (2017), 401-432. DOI: 10.1112/plms.12011 arXiv:1412.5167.

[9] A. Malheiro, "Finite derivation type for semilattices of semigroups," Semigroup Forum, vol. 84, 2012, pp. 515-526.

[10] J. M. Howie, "Fundamentals of semigroup theory," Clarendon Press, Oxford, 358. 